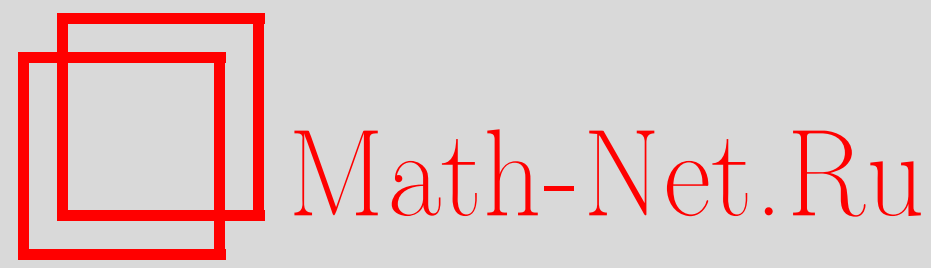

А. Ю. Васильев, Гомотопии кривых, отображений и метрика Тейхмюллера, Матем. заметки, 1996, том 59, выпуск 6, 923-926

DOI: https://doi.org/10.4213/mzm1792

Использование Общероссийского математического портала MathNet.Ru подразумевает, что вы прочитали и согласны с пользовательским соглашением

http://www . mathnet.ru/rus/agreement

Параметры загрузки:

IP : 54.205 .225 .156

26 апреля 2023 г., 09:28:21

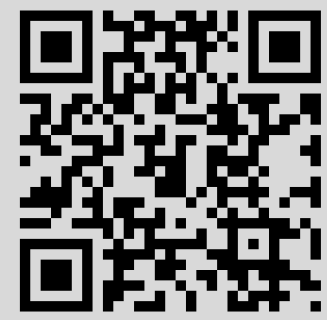




\section{ГОМОТОПИИ КРИВЫХ, ОТОБРАЖЕНИЙ И МЕТРИКА ТЕЙХМЮЛЛЕРА}

\section{А. Ю. Васильев}

Пусть $S_{0}$ - риманова поверхность конечного топологического типа $(g, n, m)$, т.е. топологически эквивалентная сфере с $g$ ручками, $n$ проколами и $m$ гиперболическими граничными компонентами. Если $f_{1}$ и $f_{2}$ - гомеоморфизмы $S_{0}$ на себя, и $f_{1}$ гомотопно $f_{2}$ в смысле гомотопии отображений, то любая замкнутая простая кривая $\gamma$ на $S_{0}$ при этих отображениях перейдет в $\gamma_{1}=f_{1}(\gamma)$ и $\gamma_{2}=f_{2}(\gamma)$, где $\gamma_{1}$ гомотопна $\gamma_{2}$ в смысле гомотопии кривых. Обратное утверждение неверно. Можно в этом убедиться на простейшем примере:

$$
S_{0}=\left\{z: r<|z|<\frac{1}{r}, 0<r<1\right\}, \quad f_{1}=z, \quad f_{2}=\frac{1}{z}, \quad z \in S_{0} .
$$

Однако в некоторых случаях можно ослабить требование гомотопии отображений, заменив его более слабым требованием гомотопии кривых. В настоящей заметке мы реализуем это утверждение при построении эквивалентного описания метрики Тейхмюллера на пространствах Тейхмюллера $T(g, n, m)$.

Пусть $(S, f)$ - отмеченная риманова поверхность, $f: S_{0} \rightarrow S$ - гомеоморфизм. Назовем $\left(S_{1}, f_{1}\right)$ и $\left(S_{2}, f_{2}\right)$ эквивалентными $(\backsim)$, если существует конформное отображение $h: S_{1} \rightarrow S_{2}$ такое, что отображение $f_{2}^{-1} \circ h \circ f_{1}$ гомотопно тождественному. Пространство Тейхмюллера $T\left(S_{0}\right)=T(g, n, m)$ есть факторпространство $\{(S, f)\} / \sim$. Пусть $x=[S]$ - точка $T\left(S_{0}\right)$, т.е. класс эквивалентности с представителем $(S, f)$. Тогда начальная точка $0=\left[S_{0}\right]$ имеет $\left(S_{0}, \mathrm{id}\right)$ своим представителем. Среди всех гомеоморфизмов $f: S_{0} \rightarrow S$ таких, что $(S, f) \in[S]=x$, гомотопных с точностью до конформного преобразования, существуют квазиконформные отображения. Обозначим через $\mu_{f}$ - комплексную дилатацию квазиконформного отображения $f$. Тогда $T\left(S_{0}\right)$ превращается в полное метрическое пространство размерности $6 g-6+2 n+3 m$ при введении метрики Тейхмюллера

$$
\tau_{T}(0, x)=\inf \frac{1}{2} \log \frac{1+\left\|\mu_{f}\right\|_{\infty}}{1-\left\|\mu_{f}\right\|_{\infty}},
$$

где нижняя грань берется по всем квазиконформным отображениям $f$ таким, что $(S, f) \in x$.

Пусть теперь $\Gamma$ - семейство гомотопических классов замкнутых жордановых кривых на $S_{0}$, не гомотопных точке или проколу, $f$ - квазиконформное отображение: $(S, f) \in[S]=x$. Определим на $S_{0}$ и $S$ проблемы модуля для $\Gamma$ и $f(\Gamma)$ в смысле П. М. Тамразова [1] (для избежания неопределенности при рассмотрении неспрямляемых квазиконформных образов жордановых кривых из $\Gamma), m(\Gamma)$ и $m(f(\Gamma))$ - модули в этих проблемах. Определим следующую величину:

$$
m_{T}(0, x)=\sup _{\Gamma} \frac{1}{2}\left|\log \frac{m(f(\Gamma))}{m(\Gamma)}\right| .
$$

Работа выполнена при поддержке Российского фонда фундаментальных исследований, грант № 95-01-00345a. 
Вообще говоря, модуль семейства нескольких гомотопических классов кривых определяется для фиксированного веса, поэтому верхнюю грань в (1) следует понимать как супремум еще и по весу.

Теорема. Величина $m_{T}(0, x)$ определяет на $T(g, n, m)$ метрику, совпадающ,ую с метрикой Тейхмюллера $\tau_{T}$.

ДоКАЗАТЕЛЬСтво проведем по этапам.

1. $m(0, x)$ конечно и определено для каждого $x$ однозначно.

Действительно, в силу гомотопических свойств $f$ и конформной инвариантности $m(\Gamma), m(f(\Gamma))$ определено одинаково для каждого семейства $Г$ независимо от $f \in[S]=x$, а, следовательно, однозначно для каждой точки $x \in T\left(S_{0}\right)$. Обозначим через $K(x)=\exp \left(2 \tau_{T}(0, x)\right)$. Тогда в силу свойств квазиконформного отображения очевидно

$$
\frac{1}{K(x)} \leqslant \frac{m(f(\Gamma))}{m(\Gamma)} \leqslant K(x)
$$

для любого $\Gamma, \mathrm{a}$, значит, существует и конечна верхняя грань в (1).

Очевидно, для $x=0 f$ гомотопно конформному отображению и $m(f(\Gamma))=m(\Gamma)$ для любого $\Gamma, m_{T}(0,0)=0$. Также очевидно неравенство треугольника: $m_{T}(0, x)+$ $m_{T}(x, y) \geqslant m_{T}(0, y)$.

2. Рассмотрим свойства дифференциалов с замкнутыми траекториями, опираясь на результаты K. Штребеля [2].

ОПРЕДЕЛЕНИЕ. Мероморфный квадратичный дифференциал $\varphi \not \equiv 0$ на римановой поверхности $S$ имеет замкнутые траектории, если незамкнутые траектории покрывают множество плоской меры 0 .

ПРЕДЛОЖЕНИЕ [2]. 1) Квадратичный дифференииал $\varphi$ с замкнутыми траекториями имеет полюса не выше второго порядка и, если $\|\varphi\|_{L_{1}}<\infty$, то полюса только первого порядка.

2) Каждая замкнутая траектория $\gamma$ для $\varphi$ с $\|\varphi\|_{L_{1}}<\infty$ вкладывается в некоторую двусвязную область $D$, ассочиированную с гомотопическим классом кривых $[\gamma]$ (т.е. каждая замкнутая кривая на S, разделяющая граничные компоненты $D$, гомотопна $\gamma$ ).

Далее будем исключать случай, когда два события появляются одновременно: $S$ - тор и $\varphi$ - голоморфньй дифференциал на торе.

ОПРЕДЕЛЕНИЕ. Пусть $\mathbb{D}=D_{j}$ некоторый набор неналегающих двусвязных областей $D_{j}$, каждая из которых ассоциированна с одним гомотопическим классом кривых $\Gamma_{j}$, где $\Gamma_{j}=[\gamma], \gamma$-замкнутая траектория дифференциала $\varphi$. Каждому гомотопическому классу $\Gamma_{j}$ соответствует единственная двусвязная область $D_{j}$ в наборе $\mathbb{D}$, ассоциированная с ним. Тогда $\mathbb{D}$ назьвается характеристическим семейством областей для дифференциала $\varphi$.

ПРЕДЛОЖЕНИЕ. 3) Для любого көадратичного мероморфного дифференциала ч с замкнутыми траекториями на $S$ с $\|\varphi\|<\infty$ существует конечное характеристическое семейство $\mathbb{D}$. (Для компактной римановой поверхности $S$ рода $g>1$ и голоморфного квадратичного дифференииала $\varphi$ количество областей в характеристическом семействе $\mathbb{D}$ не превосходит числа $3 g-3$. 
4) Пусть $\gamma-$ замкнутая траектория дифференииала $\varphi$ с замкнутыми траекториями $u\|\varphi\|<\infty, \gamma \in \Gamma_{j}$. Обозначим через $\alpha_{j}$ ее длину в метрике $|\varphi|^{1 / 2}|d z|:$

$$
\alpha_{j}=\int_{\gamma}|\varphi|^{1 / 2}|d z| .
$$

Тогда для характеристического семейства $\mathbb{D}$ справедливо неравенство

$$
\sum_{j=1}^{m} \alpha_{j}^{2} M\left(D_{j}\right) \leqslant\|\varphi\|
$$

где $M\left(D_{j}\right)$ - модуль семейства кривых, разделяющ,х граничные компоненты $D_{j}$. Равенство справедливо только для семейства $\mathbb{D}^{*}$, в котором все $D_{j}$ ограничень критическими траекториями дифференциала $\varphi$.

5) На поверхности типа $(g, n, m)$ квадратичные дифференчиаль с замкнутыми траекториями образуют всюду плотное подмножсество в пространстве квадратичных дифференциалов с конечной нормой.

3. Продолжим доказательство теоремы. Будем утверждать, что если $m_{T}(0, x)=0$, то $x=0$. Таким образом, докажем, что $m_{T}(x, y)$ - метрика. Предположим противное, т.е. $x \neq 0$. Тогда существует дифференциал Тейхмюллера $\mu=t \bar{\psi} /|\psi|, 0<t<1,\|\psi\|<\infty$, такой, что класс эквивалентности с представителем $\left(S, f^{\mu}\right)\left(f^{\mu}: S_{0} \rightarrow S\right.$ - соответствующее $\mu$ отображение Тейхмюллера) порождает точку $x \in T\left(S_{0}\right)$. Для произвольного малого $\varepsilon>0$ по предложению 5) выберем квадратичный дифференциал $\varphi$ с замкнутыми траекториями такой, что

$$
\left\|t \frac{\bar{\psi}}{|\psi|}-t \frac{\bar{\varphi}}{|\varphi|}\right\|_{\infty}<\varepsilon .
$$

Пусть $\mathbb{D}$ - характеристическое для $\varphi$ семейство областей, ассоциированных с гомотопическими классами $\Gamma=\left\{\Gamma_{j}\right\}$. Рассмотрим на $S_{0}$ проблему модуля $m(\Gamma)$, на $S=f^{\mu}\left(S_{0}\right)$ - проблему модуля $m\left(f^{\mu}(\Gamma)\right)$ и на $S_{1}=f^{\mu_{1}}\left(S_{0}\right), \mu_{1}=t \bar{\varphi} /|\varphi|,-$ проблему модуля $m\left(f^{\mu_{1}}(\Gamma)\right)=\frac{1+t}{1-t} m(\Gamma)$ (см., например, [3]). По условию $m_{T}(0, x)=0$, значит, в том числе справедливо равенство

$$
m\left(f^{\mu}(\Gamma)\right)=m(\Gamma) .
$$

Построим отображение $F: S_{1} \rightarrow S_{0} \rightarrow S$. Его комплексная дилатация есть

$$
\mu_{F}=\frac{\mu-\mu_{1}}{1-\bar{\mu} \mu_{1}} \frac{f_{\zeta}^{\mu_{1}}}{\overline{f_{\zeta}^{\mu_{1}}}}
$$

следовательно, $\left|\mu_{f}\right|<\varepsilon /\left(1-t^{2}\right)$. Обозначим $\Gamma_{1}=f^{\mu_{1}}(\Gamma), F\left(\Gamma_{1}\right)=f^{\mu}(\Gamma)$. Пусть $\mu_{0}$ - дифференциал Тейхмюллера, реализующий решение задачи Тейхмюллера о минимизации отклонения среди отображений, гомотопных $F,\left\|\mu_{0}\right\|=\varepsilon_{1}<\varepsilon /\left(1-t^{2}\right)$. Справедлива [4] вариационная формула

$$
m\left(F\left(\Gamma_{1}\right)\right)=m\left(\Gamma_{1}\right)-\varepsilon_{1} \cdot 2 \operatorname{Re} \iint_{S_{1}} \mu^{*} \varphi_{1}+o\left(\varepsilon_{1}\right),
$$


где $\mu^{*}$ - единичный вектор касательного пространства к $T\left(S_{0}\right)$ в точке $x_{1}$ с представителем $\left(S_{1}, f^{\mu_{1}}\right)$, а $\varphi_{1}$ - экстремальный квадратичный дифференциал в проблеме модуля $m\left(\Gamma_{1}\right)$. Таким образом, $m\left(F\left(\Gamma_{1}\right)\right)=m\left(f^{\mu}(\Gamma)\right)$,

$$
m\left(f^{\mu}(\Gamma)\right)-m(\Gamma)=\frac{2 t}{1-t} m(\Gamma)+\varepsilon_{1} \cdot 2 \operatorname{Re} \iint_{S_{1}} \mu^{*} \varphi_{1}+o\left(\varepsilon_{1}\right) .
$$

Так как левая часть не зависит от $\varepsilon_{1}$, то устремим $\varepsilon \rightarrow 0$. Следовательно, $\varepsilon_{1} \rightarrow 0$ и $m\left(f^{\mu}(\Gamma)\right)-m(\Gamma)>0$, что противоречит $(2)$.

3. Докажем, что полученная метрика $m_{T}$ совпадает с естественной метрикой Тейхмюллера $\tau_{T}$. Пусть $x \in T\left(S_{0}\right)$, отображение Тейхмюллера $f^{\mu}, \mu=t \bar{\varphi} /|\varphi|$, $0<t<1,\|\varphi\|<\infty$, порождает отмеченную риманову поверхность $\left(S, f^{\mu}\right)$, являющуюся представителем для $x$. Если $\varphi$-квадратичный дифференциал с замкнутьми траекториями на $S_{0}$, то в качестве $\Gamma$ рассмотрим характеристическое семейство гомотопических классов $\Gamma_{j}$; тогда на $\Gamma$ достигается верхняя грань в $(1)$ и

$$
m_{T}(0, x)=\frac{1}{2} \log \frac{1+t}{1-t}=\tau_{T}(0, x) .
$$

Если же $\varphi$ - произвольный квадратичный дифференциал на $S_{0}$ c конечной нормой, то по предложению 5) приближаем его дифференциалами с замкнутьми траекториями аналогично п. 3. Тогда верхняя грань в (1) может не достигаться, однако $m_{T}(0, x)=\tau_{T}(0, x)$. Доказательство закончено.

ЗАмЕчАниЕ 1. Мы проводим все определения и доказательства для метрик $m_{T}(0, x), \tau_{T}(0, x)$. Очевидно, эти же расстояния от произвольной точки до $x$ получаются квазиконформным сдвигом, что не нарушает общности рассуждений.

ЗАмечАниЕ 2. Для произвольного пространства Тейхмюллера величина $m_{T}(x, y)$ по п. 1 доказательства теоремы порождает полуметрику. Очевидно неравенство $m_{T}(x, y) \leqslant \tau_{T}(x, y)$.

Саратовский государственный университет

Поступило

E-mail : vasilev@scnit.saratov.su

11.07.94

\section{СПИСОК ЦИТИРОВАННОЙ ЛИТЕРАТУРЫ}

1. Тамразов П. М. Метод экстремальных метрик и конформные отображения. Автореферат дисс. ... к. ф.-м.н. Киев, 1963. 2. Strebel K. Quadratic differentials. Berlin, New York: Springer Verlag, 1984. 3. Васильев А. Ю. // Укр. матем. ж. 1991. Т. 43. № 3. С. 32-336. 4. Васильев А. Ю. // “Алгебра и анализ". Тезисы докладов международной научной конференции, посвященной 100 -летию со дня рождения Н.Г. Чеботарёва. Казань: Изд-во Казанского ун-та, 1994. С. 30. 